SLAC-PUB-11565

November 2005

\title{
Coherent States and Spontaneous Symmetry Breaking in Light Front Scalar Field Theory
}

\author{
J. P. Vary ${ }^{\mathrm{a}}$ \\ ${ }^{a}$ Iowa State University \\ Ames, Iowa 50011 \\ and \\ Lawrence Livermore National Laboratory \\ Livermore, California 94551 \\ and \\ Stanford Linear Accelerator Center \\ Stanford University, Stanford, California 94309 \\ bUniversity of Florida \\ Gainesville, Florida 32611 \\ 'Saha Institute of Nuclear Physics, Calcutta, India \\ dArkansas State University \\ State University, Arkansas 72467 \\ eInstitute of Physics \\ Bratislava, Slovakia \\ ${ }^{\mathrm{f}}$ Iowa State University \\ Ames, Iowa 50011
}

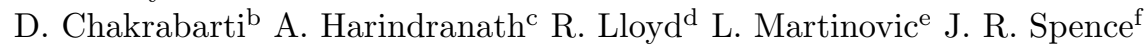

Recently developed nuclear many-body techniques provide novel results when applied to constituent quark models and to light-front scalar field theory. We show how spontaneous symmetry breaking arises and is consistent with a coherent state ansatz in a variational treatment. The kink and the kink-antikink topological features are identified and the onset of symmetry restoration is demonstrated.

\section{Introduction}

The light-front quantized Hamiltonian approach to quantum field theory has a long history. Throughout that time, Ken Wilson has been the most outspoken concerning the need to adopt successful non-relativistic microscopic many-body approaches to solve field theories nonperturbatively on the light-front [1]. Here, we outline our efforts in that direction where we capitalize on recent advances in solving the $a$ b-initio nuclear many-body problem. En route to the full quantum field theory, we solve a constituent quark model with the full treatment of color and multiple Fermions using an equal-time quantization approach. We begin with a review of the recently developed nuclear many-body techniques and proceed to the applications mentioned.

\section{Ab initio theory of nuclear structure}

In the nuclear $a b$ initio No-Core Shell Model (NCSM), we define an intrinsic "bare" Hamiltonian to include a realistic nucleon-nucleon (NN) interaction and a tri-nucleon (NNN) interaction. The NN interaction describes the $\mathrm{NN}$ data to 
high precision. These interactions may arise from any theoretical framework (meson exchange, effective field theory, chiral field theory, inverse scattering,...) and may have complicated features such as charge-symmetry breaking, non-locality, and strong repulsive behavior at short distances, among others. The NNN terms have additional parameters usually tuned to fit the properties of $A=3$ nuclei. The complexity of these Hamiltonians can easily rival that of light-front QCD in the Fermion sector.

In order to accommodate strong nuclear short-distance correlations (which generate highmomentum components), we introduce an effective Hamiltonian approach in which a 2-body or 3 -body cluster subsystem of the full $A$-body problem is solved exactly. From the exact solutions of the cluster subsystem, an effective Hamiltonian is evaluated and solved exactly [2] in a basis space appropriate to the chosen (no-core) $A$-body application.

The method involves a similarity transformation of the "bare" Hamiltonian to derive this effective Hamiltonian for a specified finite model space [3]. The $A$-body eigensolutions respect the symmetries of the underlying NN and NNN interactions. Diagonalization and the evaluation of observables from effective operators created with the same transformations are carried out on highperformance parallel computers.

For pedagogical purposes, we outline the $a b$ initio NCSM approach with NN interactions alone and point the reader to the literature for the extensions to include NNN interactions. We begin with the purely intrinsic Hamiltonian for the $A$ nucleon system, i.e.,

$$
\begin{aligned}
& H_{A}=T_{\mathrm{rel}}+\mathcal{V} \\
& =\frac{1}{A} \sum_{i<j}^{A} \frac{\left(\vec{p}_{i}-\vec{p}_{j}\right)^{2}}{2 m}+\sum_{i<j=1}^{A} V_{\mathrm{N}}(i j),
\end{aligned}
$$

where $m$ is the nucleon mass and $V_{\mathrm{N}}(i j)$, the NN interaction, with both strong and electromagnetic components. We may use either coordinate-space or momentum-space potentials.

Next, we add to (2) the center-of-mass Harmonic Oscillator $(\mathrm{HO})$ Hamiltonian $H_{\mathrm{CM}}=$ $T_{\mathrm{CM}}+U_{\mathrm{CM}}$, where $U_{\mathrm{CM}}=\frac{1}{2} A m \Omega^{2} \vec{R}^{2}, \vec{R}=$
$\frac{1}{A} \sum_{i=1}^{A} \vec{r}_{i}$. At convergence, the added $H_{\mathrm{CM}}$ term has no influence on the intrinsic properties. However, when we introduce our cluster approximation below, the added $H_{\mathrm{CM}}$ term facilitates convergence to exact results with increasing basis size. The modified Hamiltonian, with pseudodependence on the HO frequency $\Omega$, can be cast as:

$$
\begin{aligned}
H_{A}^{\Omega}= & H_{A}+H_{\mathrm{CM}}=\sum_{i=1}^{A}\left[\frac{\vec{p}_{i}^{2}}{2 m}+\frac{1}{2} m \Omega^{2} \vec{r}_{i}^{2}\right] \\
& +\sum_{i<j=1}^{A}\left[V_{\mathrm{N}}(i j)-\frac{m \Omega^{2}}{2 A}\left(\vec{r}_{i}-\vec{r}_{j}\right)^{2}\right] .
\end{aligned}
$$

We then introduce a unitary transformation, which is designed to accommodate the shortrange two-body correlations in a nucleus, by choosing an antihermitian operator $S$, acting only on intrinsic coordinates, such that

$\mathcal{H}=e^{-S} H_{A}^{\Omega} e^{S}$.

In our approach, $S$ is determined by the requirements that $\mathcal{H}$ and $H_{A}^{\Omega}$ have the same symmetries and eigenspectra over the subspace $\mathcal{K}$ of the full Hilbert space. In general, both $S$ and the transformed Hamiltonian are $A$-body operators. Our simplest, non-trivial approximation to $\mathcal{H}$ is to develop a two-body $(a=2)$ effective Hamiltonian, where the upper bound of the summations " $A$ " is replaced by " $a$ ", but the coefficients remain unchanged. We then have an approximation at a fixed level of clustering, $a$, with $a \leq A$.

$$
\begin{aligned}
\mathcal{H} & =\mathcal{H}^{(1)}+\mathcal{H}^{(a)} \\
= & \sum_{i=1}^{A} h_{i}+\frac{\left(\begin{array}{c}
A \\
2
\end{array}\right)}{\left(\begin{array}{c}
A \\
a
\end{array}\right)\left(\begin{array}{c}
a \\
2
\end{array}\right)} \sum_{i_{1}<i_{2}<\ldots<i_{a}}^{A} \tilde{V}_{i_{1} i_{2} \ldots i_{a}},
\end{aligned}
$$

with

$\tilde{V}_{12 \ldots a}=e^{-S^{(a)}} H_{a}^{\Omega} e^{S^{(a)}}-\sum_{i=1}^{a} h_{i}$,

and $S^{(a)}$ is an $a$-body operator; $H_{a}^{\Omega}=h_{1}+h_{2}+$ $h_{3}+\ldots+h_{a}+V_{a}$, and $V_{a}=\sum_{i<j}^{a} V_{i j}$. In the nuclear applications, we adopt the HO basis states that are eigenstates of the one-body Hamiltonian $\sum_{i=1}^{A} h_{i}$. In other applications, we can use any 
convenient one-body Hamiltonian and its eigenstates, usually dictated by the physical system under investigation. An example would be boxnormalized standing waves that we use in the field theory application discussed below.

The full Hilbert space is divided into a finite model space ("P-space") and a complementary infinite space (" $Q$-space"), using the projectors $P$ and $Q$ with $P+Q=1$. We determine the transformation operator $S_{a}$ from the decoupling condition $Q_{a} e^{-S^{(a)}} H_{a}^{\Omega} e^{S^{(a)}} P_{a}=0$ and the simultaneous restrictions $P_{a} S^{(a)} P_{a}=Q_{a} S^{(a)} Q_{a}=0$. The $a$-nucleon-state projectors $\left(P_{a}, Q_{a}\right)$ follow from the definitions of the $A$-nucleon projectors $P, Q$.

In the limit $a \rightarrow A$, we obtain the exact solutions for $d_{P}$ states of the full problem for any finite basis space, with flexibility for choice of physical states subject to certain conditions [4]. This approach has a significant residual freedom through an arbitrary residual $P_{a}$-space unitary transformation that leaves the $a$-cluster properties invariant. Of course, the $A$-body results are not invariant under this residual transformation. We plan to exploit this residual freedom to accelerate convergence in practical applications.

The model space, $P_{2}$, is defined by $N_{\mathrm{m}}$ via the maximal number of allowed HO quanta of the $A$ nucleon basis states, $N_{\mathrm{M}}$, where the sum of the nucleons' $2 n+l \leq N_{\mathrm{m}}+N_{\text {spsmin }}=N_{\mathrm{M}}$, and where $N_{\text {spsmin }}$ denotes the minimal possible $\mathrm{HO}$ quanta of the spectators. For ${ }^{10} \mathrm{~B}, N_{\mathrm{M}}=12, N_{\mathrm{m}}=8$ for a basis where the maximum oscillator excitation quanta is 6 . For shorthand, we refer to this as an $N_{\max }=6$ or " $6 \hbar \Omega$ " calculation. With our cluster approximation, a dependence of our results on $N_{\text {max }}$ (or equivalently, on $N_{\mathrm{m}}$ or on $N_{\mathrm{M}}$ ) and on $\Omega$ arises. The residual $N_{\max }$ and $\Omega$ dependences will infer the uncertainty in our results.

At this stage we also add the term $H_{C M}$ again with a large positive coefficient (constrained via Lagrange multiplier) to separate the physically interesting states with $0 s \mathrm{CM}$ motion from those with excited CM motion. We diagonalize the effective Hamiltonian with an m-scheme Lanczos method to obtain the $P$-space eigenvalues and eigenvectors. All observables are then evaluated free of CM motion effects. In principle, all ob- servables require the same transformation as implemented for the Hamiltonian. We obtain small renormalization effects on long range operators such as the rms radius operator and the $B(E 2)$ operator when we transform them to $P$-space effective operators at the $a=2$ cluster level $[2,5]$. On the other hand, when $\mathrm{a}=2$, substantial renormalization was observed for the kinetic energy operator [6] and for higher momentum transfer observables [5].

Recent nuclear physics applications include:

(a) spectra and transition rates in $p$-shell nuclei;

(b) role of NNN potentials from a chiral Lagrangian tied to QCD [7]

(c) comparisons between NCSM and HartreeFock [8];

(d) di-neutron correlations in the ${ }^{6} \mathrm{He}$ halo nucleus [9];

(e) neutrino cross sections on ${ }^{12} \mathrm{C}[10]$;

(f) novel NN interactions using inverse scattering theory plus NCSM [11];

(g) spectra of ${ }^{16} \mathrm{C}$ and ${ }^{16} \mathrm{O}[12]$;

(h) spectroscopy of the $A=47-49$ nuclei [13, $14]$;

(i) statistical properties of nuclei based on NCSM and approximations thereto [15].

We present an example with a soft and bare NN interaction, the Minnesota potential [16], which is a greatly simplified potential useful for testing many-body methods. The ground and first excited states of ${ }^{4} \mathrm{He}$ as a function of $\hbar \Omega$ illustrate in Fig. 1 convergence with increasing model space. Flatter and more densely packed curves signal convergence with increasing $N_{\max }$. We also find convergence for the ground state RMS radius while the RMS radius of the excited state shows divergence as one expects for a continuum state. Results obtained with $H_{e f f}$ for this same potential (not shown) are more rapidly convergent than the bare $\mathrm{H}$ results but the energies do not systematically converge from above since the variational character is absent. 


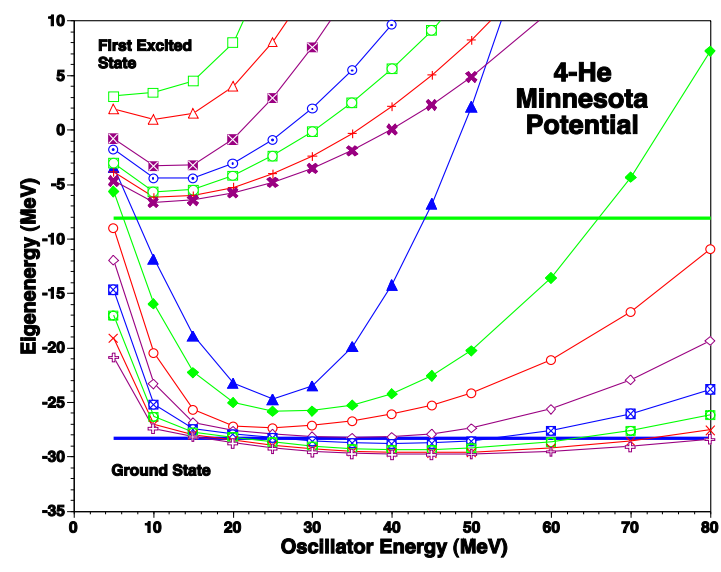

Figure 1. Ground and first excited states of ${ }^{4} \mathrm{He}$ with the Minnesota [16] bare interaction in basis spaces up to $N_{\max }=14$ as a function of $\mathrm{HO}$ energy in $\mathrm{MeV}$. The sequence of curves begins in the smallest allowed basis space and proceeds systematically down towards a flatter (more converged) result. The horizontal lines represent experiment.

\section{Constituent quark model applications}

As a prelude to working with QCD itself, we first consider a phenomenological Hamiltonian for the $q \bar{q}$ and $q q \overline{q q}$ systems [17]. We present in Fig. 2 a constituent quark model mass spectrum for three light mesons as a function of $N_{\max } / 2$. The bare Hamiltonian consists of a potential derived from a relativistic wave equation treatment motivated by QCD and supplemented with traditional assumptions of massive constituent quarks [18]. It contains a term resembling one-gluon exchange and a term with behavior close to linear confinement. The effective Hamiltonian treatment is as described above. At the $a=2$ cluster level, the effective Hamiltonian should provide the exact (convergent) result as demonstrated by the results in Fig. 2 lying on horizontal lines.

One major goal of this effort is to predict masses for exotic multiquark systems with sufficient precision to guide experimental searches as we have demonstrated for all-charm tetraquarks [17]. For this reason, all the techniques of the

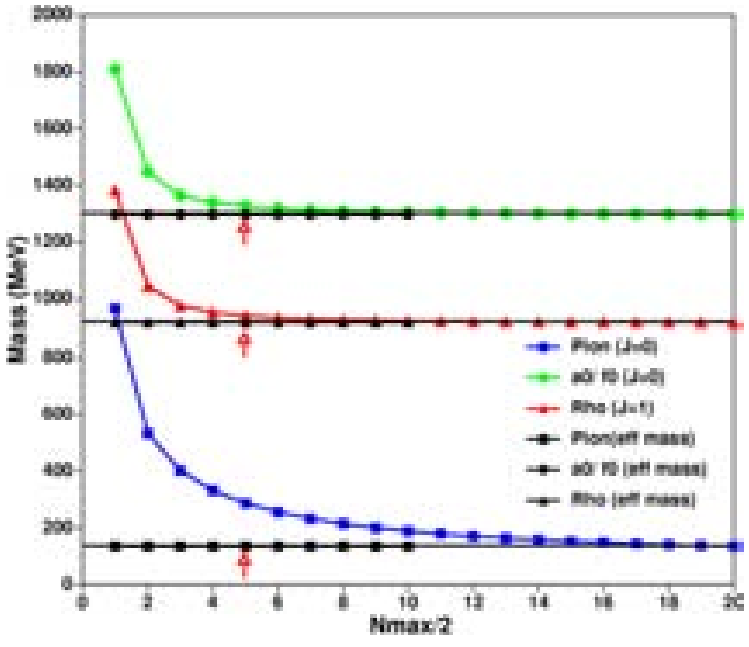

Figure 2. Three low-lying meson masses as a function of $N_{\max } / 2$. Both the bare Hamiltonian (points following curved trajectories) and the effective Hamiltonian (points following straight lines) are solved in an oscillator basis.

ab initio NCSM are needed, including the effective Hamiltonian treatment, as seen by the slow convergence of the bare Hamiltonian mass spectra with increasing basis size. Note that the inclusion of the flavor degree of freedom here is analogous to our isospin treatment in the case of nucleons. However, the introduction of color represents a major additional degree of freedom as we seek to predict global color singlet states which are antisymmetric under that exchange of color, and which lie below breakup thresholds into known mesons and baryons.

\section{Scalar field theory - spontaneous sym- metry breaking}

We discuss here selected recent results obtained with these techniques applied to light-front quantized scalar field theory in $1+1$ dimensions [1921]. For this application, we did not need to invoke the full effective Hamiltonian apparatus since it proved sufficient to work with the bare Hamiltonian for our physics goals.

Let us provide a short synopsis of this applica- 
tion and the results of three investigations for convenience. Using the Discrete Light Cone Quantization (DLCQ) Hamiltonian (box normalized plane wave basis with both periodic (PBC) [20] and anti periodic boundary condition (APBC)) $[19,21]$ we investigated non-trivial topological structures through the example of the broken symmetry phase of the two dimensional $\phi^{4}$ theory. Upon diagonalizing the bare Hamiltonian as a function of increasing basis size, we found clear evidence for degenerate ground states which is both a signature of spontaneous symmetry breaking and mandatory for the existence of kinks. Next, guided by a constrained variational calculation with a coherent state ansatz, we extracted the vacuum energy density and kink mass and compared with classical and semi - classical results. We then compared the DLCQ results for the number density of bosons in the kink state(APBC) and kink-antikink state (PBC) and obtain good agreements. Finally, we evaluated the Fourier transform of the form factor of the kink and kink-antikink and compared with the same quantity in the corresponding constrained coherent variational state and we again obtain good agreement.

Now, let us review some details of the calculations and the results. Our basis is defined by arranging massive bosons in single particle plane wave states with light front momentum $p^{+}=\frac{2 \pi n}{L}$ where $L$ is the the box length and $n$ is a half-odd integer. The many-boson states consist of all possible configurations consistent with a total lightfront momentum $P^{+}=\frac{2 \pi K}{L}$ for a chosen value of $K$. Boson number is not conserved. The even and odd particle number sectors do not mix and the signal for spontaneous symmetry breaking is the degeneracy of their lowest mass eigenstates. We show in Fig. 3 the lowest four Hamiltonian eigenvalues as a function of $1 / K$ in order to extrapolate easily to the continuum limit $K \rightarrow \infty$. Dimensions of the matrices exceed $10^{6}$.

With increasing $K$, the finite dimensional artifacts damp out. Degeneracy of the even and odd sectors with APBC is demonstrated by common trajectories of their eigenvalues and this is shown by the straight line fits in Fig. 3 (inset). Thus, the evidence for spontaneous symme-

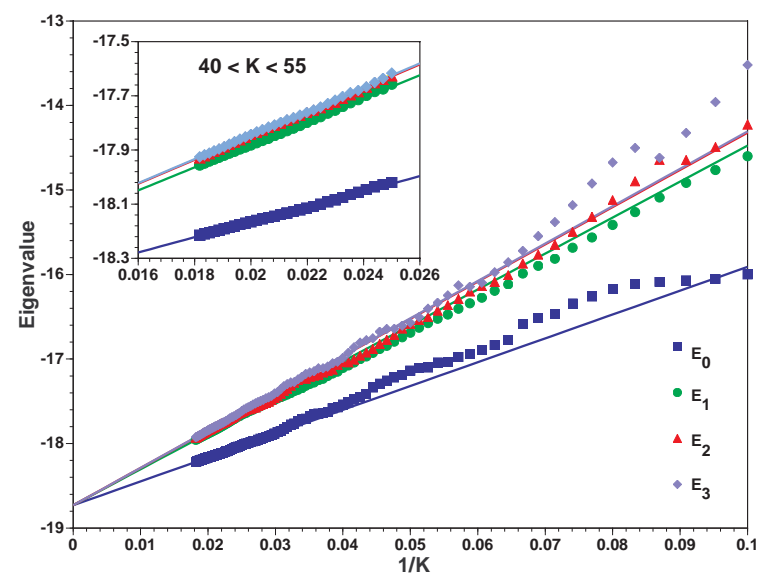

Figure 3. Lowest four eigenvalues for even and odd sectors versus $\frac{1}{K}$ for $\lambda=1.0$ with APBC. The inset shows the details over the range $40 \leq K \leq$ 55. The discrete points are the DLCQ eigenvalues while the straight lines are the linear fits to the $40 \leq K \leq 55$ data constrained to have the same intercept.

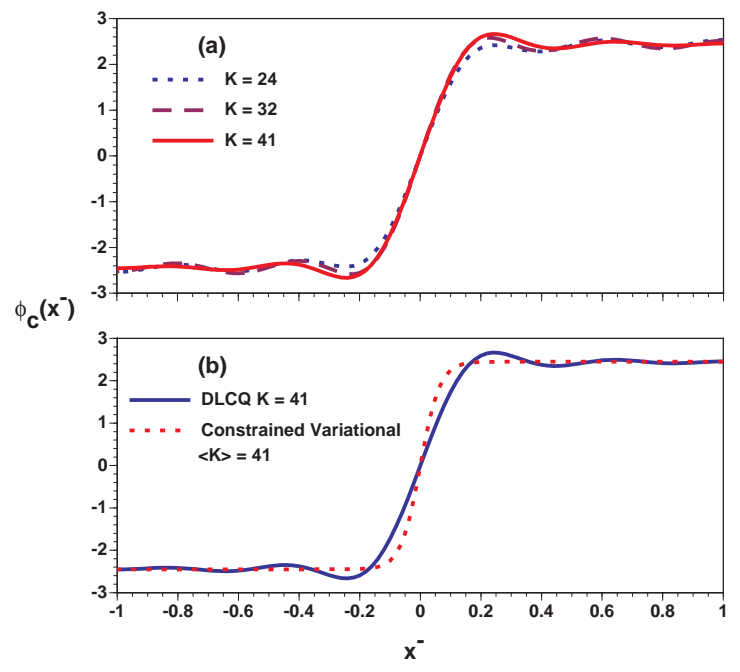

Figure 4. Fourier transform of the kink form factor at $\lambda=1$; (a) results for $K=24,32$, and 41 each obtained with DLCQ eigenstates from 11 values of $K$ centered on the designated $K$ value; (b) comparison of DLCQ profile at $\mathrm{K}=41$ with constrained variational result using the coherent state ansatz with $\langle K\rangle=41$. 
try breaking is demonstrated in this theory by our detailed non-perturbative solutions of the lightfront quantized Hamiltonian problem.

Following Rozowski and Thorn [23] who carried out an analysis with coherent states and periodic boundary condition (PBC), we evaluate the variational result for the coherent state with APBC. In addition, we include a constraint to restrict the total light front momentum, on average, to be $\mathrm{K}$. This analysis provides a viewpoint for our numerical results which is remarkably accurate. Using this coherent state analysis, we can follow the trends of our results as shown in Fig. 3 to extract the vacuum energy density and the kink mass. The fits are extrapolated to $K \rightarrow \infty$ for the kink mass (from the slope of the lowest state) and the vacuum energy density (common intercept). Results from this analysis are presented in Table 1 and provide predictions differing from those of semi-classical analyses by $10 \%$ or less over the range of coupling investigated. It would be useful to have lattice results for comparison.

\begin{tabular}{|c|cc|ccc|}
\hline$\lambda$ & \multicolumn{2}{|c|}{ vacuum energy } & \multicolumn{3}{c|}{ soliton mass } \\
\hline & CL & DLCQ & CL & SCL & DLCQ \\
\hline 0.5 & -37.70 & $-37.81(7)$ & 11.31 & 10.84 & $11.6(2)$ \\
1.0 & -18.85 & $-18.71(5)$ & 5.66 & 5.19 & $5.22(8)$ \\
1.25 & -15.08 & $-14.91(5)$ & 4.53 & 4.05 & $4.07(6)$ \\
\hline
\end{tabular}

Table 1

Comparison of vacuum energy density and soliton mass extracted from the continuum limit of our APBC DLCQ data, with classical (CL) results and, for soliton mass, the semi-classical (SCL) result [24].

Next, we compute the Fourier transform of the form factor of the lowest state which represents the kink profile[25] in the weak coupling limit. In the continuum theory,

$$
\begin{aligned}
& \int_{-\infty}^{+\infty} d q^{+} \exp \left\{-\frac{i}{2} q^{+} a\right\}\left\langle K^{\prime}\left|\Phi\left(x^{-}\right)\right| K\right\rangle \\
& =\phi_{c}\left(x^{-}-a\right) .
\end{aligned}
$$

We need the same state at different $K$ values since $K^{\prime}=K+q$. Thus, for exam-

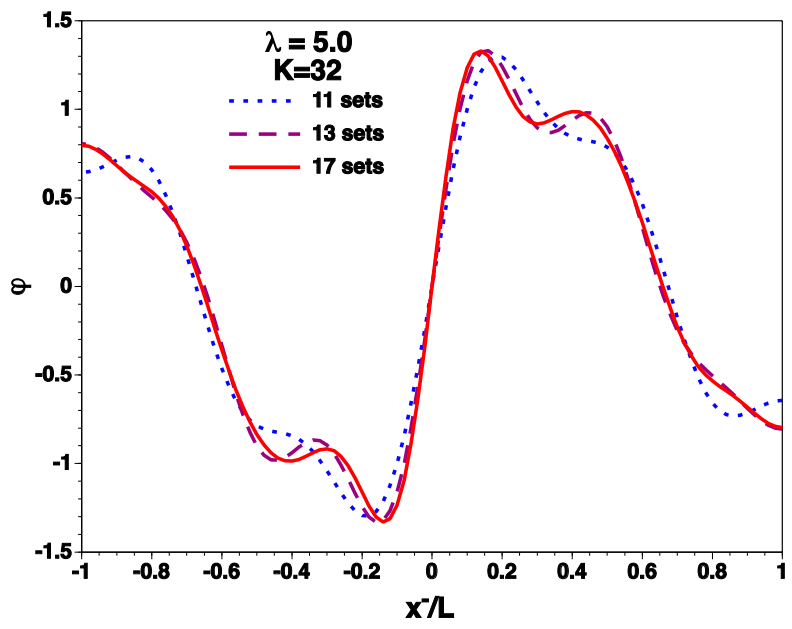

Figure 5. Fourier Transform of the kink form factor at $\lambda=5, K=32$. The figure legend indicates the number of adjoining momentum transfer terms (sets) included in the summation.

ple, we diagonalize the Hamiltonian, say, at $K=41$ in the even particle sector and at $K=$ $40.5,41.5,39.5,42.5,38.5,43.5,37.5,44.5,36.5,45.5$ in the odd particle sector. Thus, dimensionless momentum transfer ranges from -4.5 to +4.5 .

In Fig. 4 we display comparisons of our kink solutions (solid curves) with those from the constrained coherent state variational calculation. The agreement is very good.

When we examine the kink solution as a function of increasing coupling $(\lambda)$, we find evidence for the transition to a kink-antikink-kink state. This is seen for example, in the Fourier transform of the form factor for $\lambda=5$ at $K=32$ shown in Fig. 5. We expect that as we increase $K$, the steps will sharpen. Associated with this transition in the topology, the entire spectrum changes and other observables are strongly affected. The totality of changes with increased coupling indicates the onset of kink-condensation [21].

\section{Outlook}

Given the rapid progress in solving Fermionic and Bosonic systems in light-front quantized field theory, it is natural to look forward to the appli- 
cation of this non-perturbative approach to full QCD. The initial questions are the choice of basis functions and whether a renormalization program can be developed that manages the singularities that arise. We have made a promising start in this direction [22] that encourages us to forge ahead.

\section{Acknowledgments}

This work was partly performed under the auspices of the US Department of Energy by the University of California, Lawrence Livermore National Laboratory under contract No. W-7405Eng-48 and under the auspices of grants DEFG02-87ER40371, DE-AC02-76SF00515, and with support by the US National Science Foundation grant INT0137066, and by the Department of Science and Technology, India, grant DST/INT/US(NSF-RP075)/2001.

\section{REFERENCES}

1. K. Wilson, Nucl. Phys. B, Suppl. 140, $3(2005)$

2. P. Navrátil, J.P. Vary and B.R. Barrett, Phys. Rev. Lett. 84, 5728 (2000); Phys. Rev. C 62, 054311 (2000); P. Navrátil, G. P. Kamuntavičius and B. R. Barrett, Phys. Rev. C 61, 044001 (2000).

3. S. Okubo, Prog. Theor. Phys. 12, 603 (1954); J. Da Providencia and C. M. Shakin, Ann. of Phys. 30, 95 (1964); S.Y. Lee and K. Suzuki, Phys. Lett. 91B, 79 (1980); K. Suzuki and S.Y. Lee, Prog. of Theor. Phys. 64, 2091 (1980); K. Suzuki, Prog. Theor. Phys. 68, 246 (1982); K. Suzuki, Prog. Theor. Phys. 68, 1999 (1982); K. Suzuki and R. Okamoto, Prog. Theor. Phys. 70, 439 (1983).

4. C.P. Viazminsky and J.P. Vary, J. Math. Phys., 42, 2055 (2001).

5. I. Stetcu, B.R. Barrett, P. Navrátil and J. P. Vary, Phys. Rev. C 71, 044325(2005); I. Stetcu, B.R. Barrett, P. Navrátil and C.W. Johnson, to be published; nucl-th/0409072.

6. H. Kamada, et. al, Phys. Rev. C 64044001 (2001).

7. A. Nogga, E. Epelbaum, P. Navrátil, W., Glockle, H. Kamada, Ulf-G. Meißner, H.
Witala, B.R. Barrett, and J.P. Vary, Nucl. Phys. A737, 236 (2004); A. Nogga, P. Navrátil, B.R. Barrett and J. P. Vary, in preparation; P. Navrátil, J. P. Vary, V. G. Gueorguiev and W. E. Ormand, in preparation.

8. M.A. Hasan, J.P. Vary, and P. Navrátil, Phys. Rev. C 69, 034332 (2004).

9. O. Atramentov, J.P. Vary and P. Navrátil, in preparation.

10. A. C. Hayes, P. Navrátil and J. P. Vary, Phys. Rev. Lett. 91, 012502 (2003).

11. A.M. Shirokov, A.I. Mazur, S.A. Zaytsev, J.P. Vary and T.A. Weber, Phys. Rev. C 70, 044005 (2004); A.M. Shirokov, J. P. Vary, A.I. Mazur, S.A. Zaytsev and T.A. Weber,Phys. Letts. B 621, 96(2005); and to be published.

12. P. Navrátil and J. P. Vary, in preparation.

13. S. Popescu, S. Stoica, J.P. Vary and P. Navrátil, submitted for publication.

14. A. Negoita, S. Stoica, J.P. Vary and P. Navrátil, in preparation.

15. B. Shehadeh and J.P. Vary, in preparation.

16. D. R. Thompson, M. LeMere and Y. C. Tang, Nucl. Phys. A 286, 53(1977); I. Reichstein and Y. C. Tang, Nucl. Phys. A 158, 529(1970).

17. R.J. Lloyd and J.P. Vary, Phys. Rev. D 70, 014009 (2004); R.J. Lloyd, J.R. Spence and J.P. Vary, to be published.

18. J.R. Spence and J.P. Vary, Phys. Rev. C 59, 1762 (1999); and to be published.

19. D. Chakrabarti, A. Harindranath, L. Martinovic and J.P. Vary, Phys. Lett. B 582, 196 (2004).

20. D. Chakrabarti, A. Harindranath, L. Martinovic G. Pivovarov and J.P. Vary, Phys. Lett. B 617, 92 (2005).

21. D. Chakrabarti, A. Harindranath and J.P. Vary, Phys. Rev. D 71, 125012(2005).

22. D. Chakrabarti, A. Harindranath and J.P. Vary, Phys. Rev. D 69, 034502 (2004);

23. J. S. Rozowsky and C. B. Thorn, Phys. Rev. Lett. 85, 1614 (2000).

24. R. F. Dashen, B. Hasslacher and A. Neveu, Phys. Rev. D 10, 4130 (1974).

25. J. Goldstone and R. Jackiw, Phys. Rev. D 11, 1486 (1975). 